$4^{\text {th }}$ International Conference on Industrial Engineering and Industrial Management

XIV Congreso de Ingeniería de Organización

Donostia - San Sebastián, September $8^{\text {th }}-10^{\text {th }} 2010$

\title{
Evaluación del impacto del reprocesado en los sistemas Kanban y Conwip*
}

\section{Assessing the impact of reprocessing in Kanban and Conwip systems}

\author{
Pedro L. González-R., José M. Molina, José M. León y Rafael Ruiz-Usano \\ Dpto. de Ingeniería Civil. Área de Organización de Empresas. \\ Dpto. de Organización Industrial y Gestión de Empresas. Escuela Superior de Ingenieros. Universidad de Sevilla. \\ Camino de los Descubrimientos s/n, 41092 Sevilla. \\ pedroluis@esi.us.es, jmolinap@esi.us.es, miguel@esi.us.es, usano@esi.us.es.
}

Fecha de recepción: 8-9-2010

Fecha de aceptación: 27-10-2010

Resumen: Desde las primeras implementaciones de los sistemas de control de la producción basados en tarjetas (kanbans) han ido evolucionando (apareciendo nuevos sistemas) y aplicándose paulatinamente en la práctica. A medida que crece su uso en diferentes entornos, han surgido dudas acerca de la selección de uno u otro sistema dependiendo de las características particulares del entorno. En este sentido se enmarca el presente trabajo, centrándonos en el comportamiento de los sistemas Kanban y Conwip bajo el reprocesado de piezas en una estación. Los resultados muestran la conveniencia del empleo del sistema Kanban con respecto al Conwip.

Palabras clave: Conwip, Kankan, Reprocesado.

Abstract: From the first implementations of card based (kanbans) production control systems, these have evolved, (appearing new systems) and have been gradually applied in practice. As its use grows in different environments, there have been doubts about the selection of one or the other depending on the features of the environment. In this sense this work is framed, focusing on the behavior of Kanban and Conwip systems under jobs rework in a station. The results show the suitability of the use of a Kanban system with respect to Conwip.

Keywords: Conwip, Kankan, Rework.

\section{Introducción}

Los sistemas de control de la producción basados en tarjetas, como el Conwip (Spearman et al., 1990) y el Kanban (Sugimory et al., 1977) han demostrado ser aplicables a una gran variedad de entornos de fabricación, incluyendo entornos de flujo lineal, talleres, líneas de montaje, o incluso a sistemas más complejos como son la propia coordinación de la cadena de suministro (ver por ejemplo Hopp 2007, o Xiaobo et al. 2007).

Sin embargo, que sepamos, no existen reglas claras sobre la aplicabilidad de estos sistemas de control de la producción a escenarios concretos (detalles sobre la aplicabilidad de los sistemas Conwip pueden encontrarse en Framinan et al. 2003). Uno de los entornos en donde ha sido aplicado es bajo reprocesado (ver Duri et al., 2000), pero no se dan indicios sobre el impacto que el porcentaje de trabajos defectuosos tiene en el rendimiento del sistema, sino que dicho estudio ha sido enfocado al ajuste del número de tarjetas que obtiene el mejor resultado esperado de acuerdo a funciones de costes predefinidas. Sin este «análisis de sensibilidad», la aplicación es poco más que un ejercicio y no proporciona información valiosa para su aplicación a nivel práctico.

\footnotetext{
* Los resultados de este trabajo han sido financiados por el Ministerio de Ciencia e Innovación a través del proyecto «SAGIP: Sistemas Avanzados para la Gestión Integrada de Pedidos» (DPI2007-6I345) y el proyecto «SCOPE: Sistemas Cooperativos para la programación y ejecución de pedidos» (P08-TEP-3630) financiado por la Junta de Andalucía.
} 
Hay que tener en cuenta que la mayor parte de estudios sobre reprocesado se centran en la definición de políticas adecuadas de inspección y no en el impacto del reprocesado sobre el propio rendimiento del sistema. La necesidad de volver a procesar una pieza en un sistema de control de la producción basado en tarjetas tiene un efecto que no aparece en la aparición de otros eventos adversos como la aparición de averías o como la necesidad de realizar un cambio de partida (set-up). Nos referimos al hecho de que una pieza que es necesario reprocesar altera el flujo normal de las tarjetas, por lo que afecta al rendimiento global del sistema (Framinan et al., 2003).

En este trabajo se trata de determinar la influencia del reprocesado en dos sistemas de control de la producción con enorme repercusión práctica, como son el sistema Conwip y Kanban. En la sección 2 se introduce brevemente la operativa de estos sistemas. En la sección 3 se detallan los escenarios sobre los que se ha conducido la experimentación. En la sección 4 se muestran y discuten los resultados. Finalmente en la sección 5 se indican las conclusiones del estudio y se aportan futuras líneas de actuación.

\section{I. Descripción de los sistemas}

\section{Sistema Kanban}

Sugimori et al. (1977) proporcionan una de las primeras descripciones del sistema de Kanban, y se pueden encontrar revisiones de dichos sistemas Kanban en Uzsoy y Martin-Vega (1990), Berkley (1992), Akturk Erhun (1999), y recientemente en Lage y Godinho $(20 \mid 0)$.

Los sistemas Kanban se basan en un mecanismo de bloqueo que depende de la capacidad máxima del buffer. Habitualmente suelen describirse dos tipos de implementaciones de este sistema: Kanban Simple (individual) o Kanban Doble. Ambos tipos de sistemas emplean tarjetas para controlar el flujo de materiales en las estaciones. La diferencia básica entre ambos radica en el proceso de transporte entre estaciones, el cual se supone instantáneo en el Kanban Simple y controlado por tarjetas en el Kanban Doble. Sin pérdida de generalidad, en nuestro estudio emplearemos el sistema Kanban Simple, cuya operativa se describe brevemente a continuación.

En este sistema, el inventario en proceso de cada estación debe ser menor o igual que una cierta cantidad, usualmente cuantificada a través de tarjetas, $k_{i}$. Cada vez que un trabajo (pieza) entra en un buffer a la entrada de una estación, se le asocia una tarjeta a éste, la cual se permanece vinculada a él hasta que el trabajo sale de la estación. Cada estación podrá procesar nuevos trabajos siempre que disponga de tarjetas libres que todavía no hayan sido asociadas a trabajos. Este mecanismo también se conoce como Instantaneous Material Handling (ver Berkley, 1992) o Immediate Material Transfer (Gstettner y Kuhn, 1996). Los parámetros para cada estación en este sistema Kanban son el número de tarjetas en cada estación. La Figura I muestra el flujo de trabajos y tarjetas en una línea de cinco estaciones controlada por un sistema Kanban Simple.

\subsection{Sistema Conwip}

El sistema Conwip (Constant Work In Process) basa su control sobre el proceso manteniendo constante el inventario en proceso (véase Spearman et al. 1990 para más información sobre el sistema). En este sistema, se asocia una tarjeta a un trabajo al principio de la línea de fabricación. De esta manera el máximo número de trabajos en la línea coincide con el número de tarjetas disponibles inicialmente en el sistema. Cuando un trabajo ha terminado su proceso, la tarjeta adjunta se libera y se envía al principio de la línea, donde queda disponible para ser asociada a trabajo.

Por tanto, para su funcionamiento, es sólo necesario emplear un parámetro, el número de tarjetas inicia-

Figura I

Sistema Kanban Simple

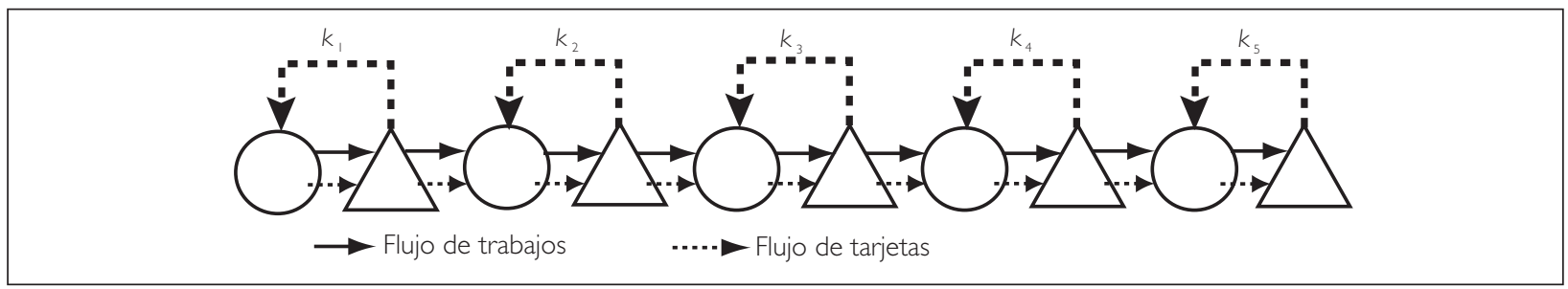

Dirección y Organización, Núm. 42, Diciembre 2010 | ISSN (On line): 2171-6323 - ISSN (Print): II32-I75X 
Figura 2

Sistema Conwip

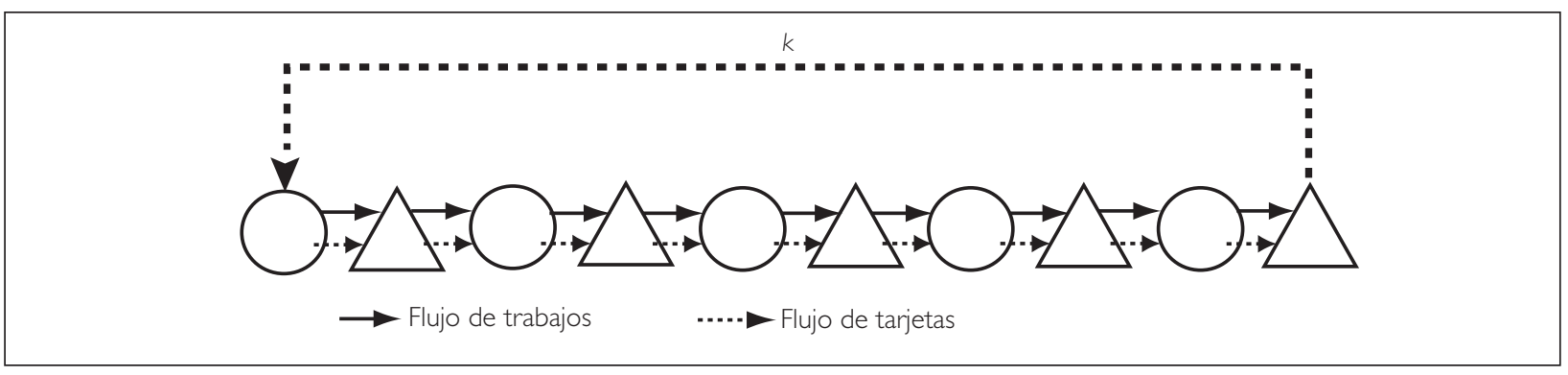

les, $k$. En la Figura 2 se muestra una línea Conwip de cinco estaciones en serie.

Hay dos aspectos en los que existe un poco de controversia respecto el funcionamiento de estos sistemas. El primero es respecto a si puede ser interpretado como un sistema push o pull. Aunque clásicamente el sistema Conwip se considera de tipo pull (por su relación con el sistema Kanban, clásicamente definido como pull), algunos autores consideran que tiene características de ambos tipos de sistemas (véase por ejemplo, Pyke y Cohen, 1990). El segundo es si funciona mejor o peor que otros sistemas. Por lo general, el funcionamiento de los sistemas depende del tipo de entorno (escenario) en donde se aplique. Por lo tanto, no es posible (o es muy complicado) obtener resultados generales que puedan ser extrapolables a otros escenarios. Este aspecto nos parece de interés y centra gran parte de nuestro trabajo. Para una discusión detallada sobre este tema, véase la revisión de Framinan et al. (2003). Sin embargo, el sistema Conwip se ha utilizado como un sistema de referencia para comparar el desempeño de otros nuevos sistemas, ya que el sistema Conwip ha mostrado un buen comportamiento en una gran variedad de escenarios.

\section{Experimentación}

La experimentación que aquí se presenta se ha diseñado con el objeto de realizar un análisis sobre el funcionamiento de los sistemas Conwip y Kanban ante un escenario de reprocesado. Se ha partido de una línea formada por cinco estaciones. Este número nos parece adecuado ya que la mayor parte de los estudios de reprocesado se han centrado en la aplicación a una máquina (ver por ejemplo Agnihothri y Kenett 1995, Cárdenas-Barrón 2007, o Barketau et al., 2008), aunque es posible encontrar aplicaciones de hasta tres (ver por ejemplo Duri et al., 2000).
Se ha considerado que dicha línea está equilibrada en cuanto a tiempos de proceso. Sin embargo este equilibrio se verá alterado por la aparición de fenómenos o eventos adversos como son la consideración de reprocesados. El reprocesado se considerará en la estación central de manera que una pieza defectuosa deba ser procesada de nuevo en la misma estación. Se estudian diferentes niveles del mismo, de acuerdo a un parámetro que hemos denominado porcentaje de reprocesado, $P R$ : $\{0 \%, 5 \%$, $10 \%\}$.

El tiempo de proceso se considera no determinista, con el objeto de acercar el modelo a un sistema cercano a la realidad. Además se han considerado diferentes situaciones en cuanto a la dispersión de los tiempos de proceso, lo cual nos permite abordar una amplia gama de situaciones. Como se argumenta en Hopp y Spearman (2000), los sistemas con un coeficiente de variación (cv) cercanos a cero suelen ser habituales en sistemas con un alto nivel de automatización. Por otra parte, sistemas con un coeficiente de variación superiores a la unidad suelen ser poco realistas. En nuestra experimentación, con el objeto de abordar todas las situaciones anteriores, se han estudiado diferentes niveles de dispersión, para los siguientes valores del coeficiente de variación, $c v:\{0.1,0.5,0.9\}$. Partiendo de un valor medio del tiempo de proceso $\bar{p}_{\mathrm{\imath}}$ para la estación i, para un determinado cv, el tiempo de proceso en cada estación se genera de acuerdo a una distribución gamma $\Gamma(k, \theta)$ con un factor de forma $k=1 / c v^{2}$ y un factor de escala $\theta=c v^{2} \bar{p}$. Por tanto, el tiempo de proceso en cada estación $p_{\mathrm{u}}$, es generado de acuerdo a una distribución $\Gamma\left(\mid / \mathrm{CV}^{2}, C v^{2}\right.$ $\bar{p})$. En el estudio realizado, al considerar el sistema inicialmente equilibrado se ha considerado que $\bar{p}_{\iota}=3, \forall i=1,2, \ldots, 5$.

De manera esquemática los sistemas estudiados se representan en las siguientes figuras: 
Figura 3

Sistema Kanban bajo reprocesado

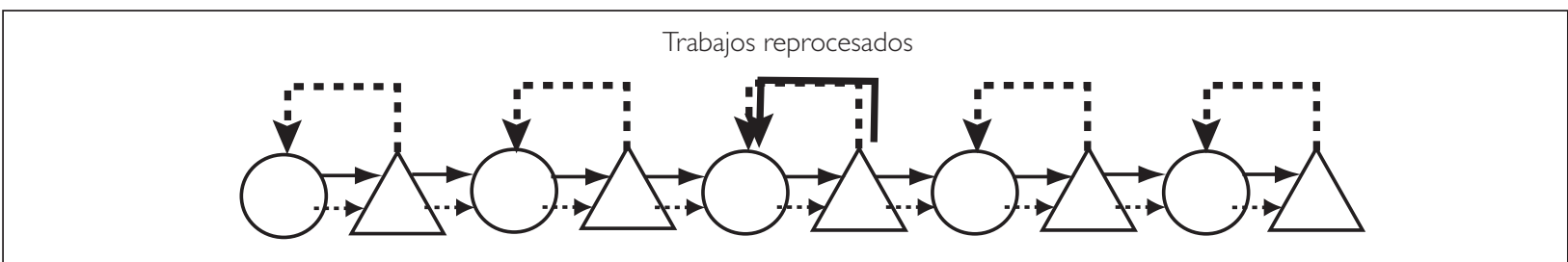

Figura 4

Sistema Conwip bajo reprocesado

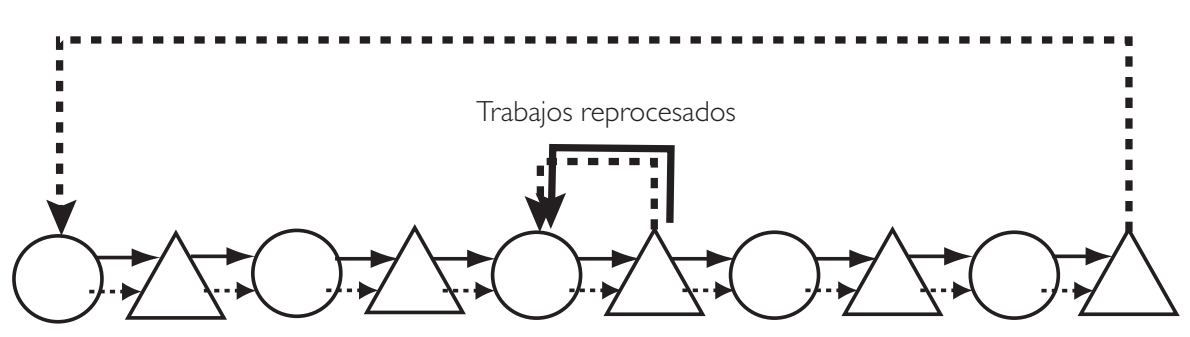

Hay que tener en cuenta que tanto el sistema Conwip como el Kanban ejercen el control del flujo de piezas mediante tarjetas. Sin embargo el sistema Conwip sólo necesita definir el número de tarjetas a emplear, k, mientras que el sistema Kanban realiza el control de manera individual en cada estación y no de manera conjunta como el sistema Conwip. Por tanto necesita definir un número de tarjetas para cada estación, siendo el parámetro de control, un vector $\bar{k}:\left\{k_{1}, k_{2}, \ldots, k_{v}\right\}$, siendo $n$ el número de estaciones en la línea. Obviamente el funcionamiento de ambos sistemas varía dependiendo del número de tarjetas asignadas al sistema. Para realizar una comparación exhaustiva sobre el funcionamiento de ambos sistemas, sería necesario explorar todas las posibles soluciones. Una solución vendrá determinada por el número de tarjetas $k$ en el sistema Conwip o por el conjunto de número de tarjetas a través del vector k para el sistema Kanban. Considerando un número de tarjetas máximo $N_{\mu \alpha \xi}$, el espacio de soluciones para el sistema Conwip sería $N_{\mu \alpha \xi}$, pero para el sistema Kanban, para un sistema con $n$ estaciones ven-

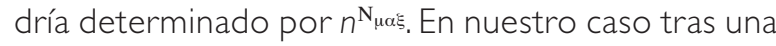
experimentación previa se ha tomado un valor de $N_{\mu \alpha \xi}=30$. Esto hace que el espacio de soluciones para el sistema Kanban sea superior a 9 • 3 • $10^{20}$. A esto hay que sumarle el hecho de que al considerar estocasticidad en los tiempos de proceso, sea necesario realizar varios replicados con el objeto de poder determinar la dispersión de los resultados. Por tanto al tratarse de un elevado número de simulaciones, la opción de una enumeración exhaustiva para el sistema Kanban queda descartada. En dicho caso parece conveniente emplear algún método de optimización. Para ello se ha decidido modelar el sistema en ARENA 12, y emplear el optimizador OPTQUEST que incluye el mismo paquete, lo cual es una técnica habitual en este tipo de trabajos. Un análisis previo nos ha permitido establecer el horizonte de simulación en unidades de tiempo y 5 replicados.

Con el objeto de poder comparar ambos sistemas ante una misma situación se ha decidido establecer una tasa de salida mínima, establecida a 0.3 [piezas / unidad de tiempo], lo que constituye establecer la tasa de salida mínima al $90 \%$ de la capacidad de la línea. Por tanto, se compararán los sistemas principalmente en base al WIP obtenido. A modo de resumen se muestra en la Tabla I los factores intervinientes en el experimento y los valores que pueden tomar.

Tabla I

Diseño experimental

\begin{tabular}{|c|c|}
\hline Factor & Niveles \\
\hline Sistema & Conwip, Kanban \\
\hline Coeficiente de Variación cV & $0.1,0.5,0.9$ \\
\hline Porcentaje de reprocesado PR (\%) & $0,5,10$ \\
\hline
\end{tabular}


El diseño experimental llevado a cabo es un diseño factorial completo, por lo que se han ejecutado un total de $2 \cdot 3^{2}=18$ experimentos, o lo que es lo mismo 9 escenarios de optimización para cada sistema. Los resultados obtenidos se muestran en la siguiente sección.

\section{Resultados}

Los resultados experimentales se muestran en la Tabla 2. En dicha tabla se manifiestan para cada uno de los 18 escenarios estudiados los valores del WIP y tasa de salida $T H$, esta última medida en tanto por ciento respecto a la capacidad del sistema. Para ambos valores de respuestas se muestra además el valor del Intervalo de Confianza (IC), para un nivel de significación del 95\%. Finalmente se muestra el valor del número de tarjetas para las que el optimizador
OPTQUEST ha encontrado un menor WIP, pero alcanzando una $\mathrm{TH}$ superior o igual al objetivo mínimo fijado del $90 \%$.

Con el objeto de estudiar la influencia de los diferentes factores sobre las respuestas se ha realizado un análisis ANOVA aplicando la técnica de pooling (ver Montgomery 2002 para más detalles). Los resultados para el WIP y el TH se muestran en las tablas 3 y 4 respectivamente, indicando con un asterisco aquellos factores que resultaron significativos en el estudio.

Del análisis ANOVA se deriva que el sistema Kanban y Conwip obtienen un resultado significativamente distinto, lo cual es el principal resultado del estudio. Por otra parte hay que tener en cuenta que el cr y el PR son factores que afectan significativamente al WIP. Respecto al nivel de TH alcanzado, no existen

Tabla 2

Resultados

\begin{tabular}{|c|c|c|c|c|c|c|c|c|c|c|c|c|c|c|c|}
\hline \multirow[b]{3}{*}{$\mathrm{cr}$} & \multirow[b]{3}{*}{$\operatorname{PR}(\%)$} & \multicolumn{14}{|c|}{ Sistema } \\
\hline & & \multicolumn{5}{|c|}{ Conwip } & \multicolumn{9}{|c|}{ Kanban } \\
\hline & & WIP & $\mathrm{Cl}(95 \%)$ & $\mathrm{TH}(\%)$ & $\mathrm{Cl}(95 \%)$ & $k$ & WIP & $\mathrm{Cl}(95 \%)$ & $\mathrm{TH}(\%)$ & $\mathrm{Cl}(95 \%)$ & $k_{1}$ & $k_{2}$ & $k_{3}$ & $k_{4}$ & $k_{5}$ \\
\hline \multirow{3}{*}{0,1} & 0 & 5,00 & 0,0000 & 92,83 & 0,0320 & 5 & 4,80 & $0,00 \mid 4$ & 90,93 & 0,0460 & I & 1 & 1 & I & 1 \\
\hline & 5 & 6,00 & 0,0000 & 93,61 & 0,3020 & 6 & 5,48 & 0,0122 & 92,51 & 0,1429 & । & I & 2 & । & 6 \\
\hline & 10 & 33,00 & 0,0000 & 90,01 & 0,1569 & 33 & 7,81 & 0,0054 & 90,01 & 0,3731 & 2 & I & 3 & । & 4 \\
\hline \multirow{3}{*}{0,5} & 0 & 12,00 & 0,0000 & 90,17 & 0,1805 & 12 & 10,67 & 0,2009 & 90,68 & 0,2415 & I & 3 & 5 & 3 & 7 \\
\hline & 5 & 15,00 & 0,0000 & 90,75 & 0,4346 & 15 & 13,08 & 0,1227 & 90,55 & 0,2859 & 2 & 2 & 7 & 14 & 20 \\
\hline & 10 & 29,00 & 0,0000 & 90,00 & 0,5854 & 29 & 19,86 & $0,09 \mid 1$ & 90,03 & $0,50 \mid 4$ & । & 5 & II & 10 & 19 \\
\hline \multirow{3}{*}{0,9} & 0 & 29,00 & 0,0000 & 90,07 & 0,2285 & 29 & 23,64 & 0,8955 & 90,25 & $0,607 \mid$ & I & 8 & 10 & 15 & 20 \\
\hline & 5 & 35,00 & 0,0000 & 90,15 & 0,7628 & 35 & 30,01 & 0,5640 & 90,19 & 0,4885 & 5 & 8 & 9 & 9 & 13 \\
\hline & 10 & 59,00 & 0,0000 & 90,11 & 0,7368 & 59 & 37,80 & 0,4267 & 90,02 & 0,9482 & । & 15 & 16 & 14 & 19 \\
\hline
\end{tabular}

Tabla 3

ANOVA factorial para WIP, $\alpha=0.1$

\begin{tabular}{|c|c|c|c|c|c|c|}
\hline Source & SS & DoF & MS & F-Ratio & Sign. & p-value \\
\hline Sistema & 264.30 & 1 & 264.30 & 13.71 & $*$ & 0.021 \\
\hline cv & 1660.80 & 2 & 830.40 & 43.08 & $*$ & 0.002 \\
\hline PR & 769.60 & 2 & 384.80 & 19.96 & $*$ & 0.008 \\
\hline Sistema $\times c v$ & 24.86 & 2 & 12.43 & 0.65 & & 0.572 \\
\hline Sistema $\times$ PR & 155.38 & 2 & 77.69 & 4.03 & & 0.110 \\
\hline C $\times$ PR & 18.16 & 4 & 4.54 & 0.24 & & 0.905 \\
\hline Pooled & 77.12 & 4 & 19.28 & & & \\
\hline TOTAL & 2970.22 & 17 & & & & \\
\hline
\end{tabular}


Tabla 4

ANOVA factorial para $\mathrm{TH}, \alpha=0 . \mathrm{I}$

\begin{tabular}{|c|c|c|c|c|c|c|}
\hline Source & SS & DoF & MS & F-Ratio & Sign. & P-value \\
\hline Sistema & 0.728 & 1 & 0.728 & 2.063 & & 0.181 \\
\hline cV & 8.911 & 2 & 4.456 & 12.63 & $*$ & 0.002 \\
\hline PR & 3.203 & 2 & 1.602 & 4.539 & $*$ & 0.040 \\
\hline C $\times$ PR & 3.952 & 2 & 1.976 & 5.601 & $*$ & 0.023 \\
\hline Pooled & 3.528 & 10 & 0.3528 & & & \\
\hline TOTAL & 20.32 & 17 & & & & \\
\hline
\end{tabular}

diferencias significativas entre ambos sistemas. Esto tiene cierto sentido, ya que una de las restricciones impuestas en el estudio era alcanzar una tasa de salida del $90 \%$, por lo que los sistemas se han intentado ajustar a dicho valor. Sin embargo, igual que ocurría en elWIP, tanto el cv como el PR y su interacción $C V \times P R$ resultan significativas, es decir el TH es sensible a nivel de $c$ y $P R$.

Sin embargo del análisis anterior no se puede extraer ninguna conclusión acerca de la evolución de las respuestas respecto a los diferentes niveles de los factores. Para poder valorar dichos efectos en la respuesta de interés (WIP) se muestran a continuación las gráficas de medias marginales tanto para los niveles del cv (Figura 5) y PR (Figura 6) y para cada sistema objeto de estudio.

Figura 5

Gráfica de Medias Marginales (WIP vs Sistema vs cv)

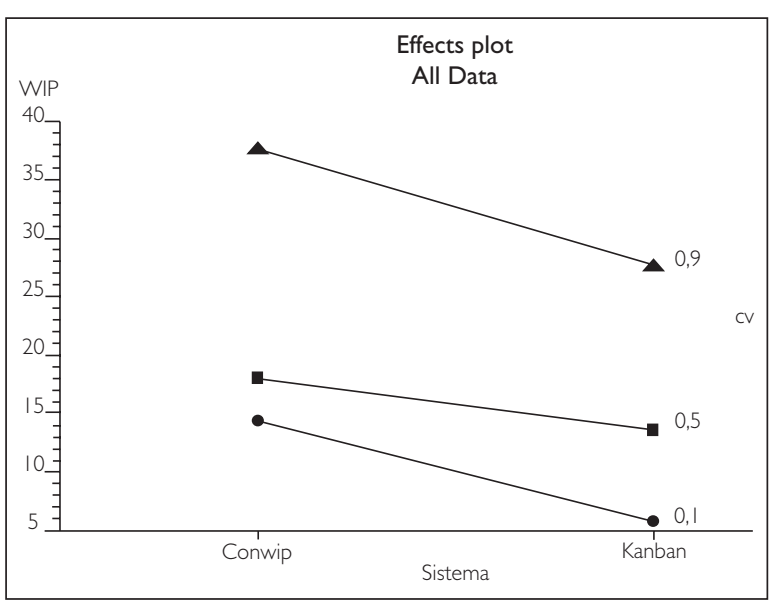

En esta gráfica se puede observar que a medida que aumenta el cv, también aumenta el WIP. Por otra parte se puede observar que el sistema Kanban obtiene en promedio un valor de WIP inferior al sistema Conwip. Se puede comprobar en la Tabla 2 de re- sultados que este efecto se produce en todos los escenarios, bajo las mismas condiciones. Como es de esperar el funcionamiento es similar en el caso de que no exista reprocesado, aunque es conocido que en sistemas equilibrados el sistema Kanban mejora al Conwip.

Figura 6

Gráfica de Medias Marginales (WIP vs Sistema vs PR)

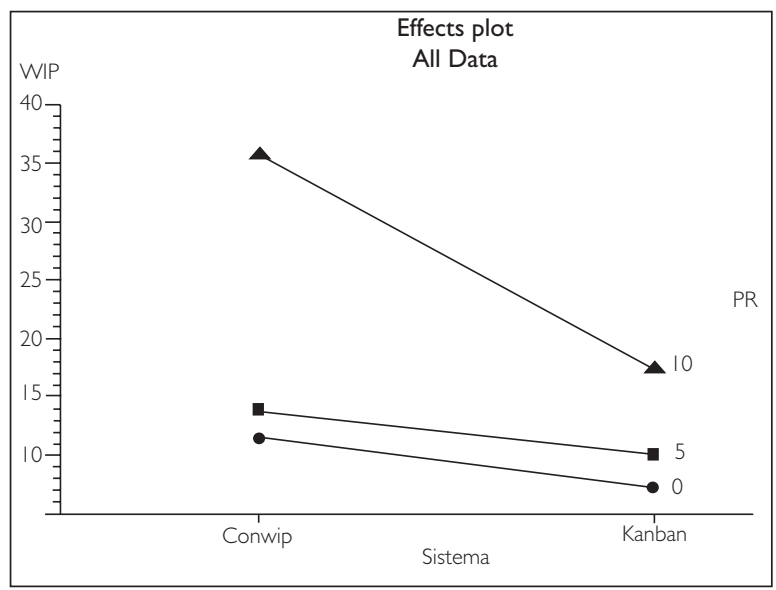

Con respecto al procesado los valores promedios indican el mismo efecto, es decir, por una parte un peor funcionamiento de ambos sistemas a medida que el $P R$ se incrementa y un peor funcionamiento del sistema Conwip respecto al Kanban.

Las anteriores gráficas, sin embargo no permiten evaluar la influencia conjunta de los efectos del cr y PR en ambos sistemas, la cual se puede interpretar mejor mediante una gráfica de contorno, como la mostrada en la Figura 7. Se puede observar que el deterioro del sistema Conwip se produce para cualquier coeficiente de variación, de manera creciente con la cantidad de reprocesados. Ante una combinación de variabilidad (elevado cv) y gran cantidad de reprocesados, el 
sistema Conwip alcanza los peores resultados. Por el contrario, el sistema Kanban ofrece un comportamiento mucho más estable incluso para las situaciones más extremas. Parece por tanto que el efecto es mucho más perjudicial en el sistema Conwip.

Dichos resultados coinciden con las observaciones realizadas en el ANOVA, donde se manifiesta que el c $v$ de los tiempos de proceso afecta en mayor medida a la respuesta del sistema que el PR. También cabe resaltar la interacción entre ambos efectos, mucho más perjudicial en el sistema Conwip que en el sistema Kanban.

\section{Conclusiones}

En el presente trabajo se ha puesto de manifiesto el empeoramiento del funcionamiento de un sistema de control de la producción tipo Conwip, comparado con el sistema Kanban en el escenario objeto de estudio, bajo la influencia de reprocesado y estocasticidad de los tiempos de proceso. Como muestran los resultados, la alteración en el flujo de las tarjetas produce en el sistema Conwip un empeoramiento que se muestra en el incremento en el WIP. Sin embargo el escenario estudiado es muy limitado y sería necesario completar este estudio a otros escenarios más detallados, que incluyan diferentes tasas de salida objetivo y diferentes patrones de desequilibrio en la línea. Por otra parte sería interesante estudiar la influencia del reprocesado en otros entornos de aplicación más cercanos a la realidad, como en estaciones de montaje o en entornos de tipo taller.

\section{Referencias}

AGNIHOTHRI, SR y KENETT, RS. ( 1995). «The impact of defects on a process with rework». European Journal of Operational Research, 80 (2), pp. 308-327.

AKTURK, M.S. y ERHUN, F. (1999). «An overview of design and operational issues of kanban systems», International Journal of Production Research, 37 (17), pp. 3859-3881.

BARKETAU, MS; CHENG, TCE y KOVALYOV, MY. (2008). «Batch scheduling of deteriorating reworkables», European Journal of Operational Research, I 89 (3), pp. I 317 1326.

BERKLEY, B.J. ( 1992). «A review of the kanban production control research literature», Production and Operations Management, I (4), pp. 393-4II.

CÁRDENAS-BARRÓN, LE. (2007). «On optimal manufacturing batch size with rework process at single-stage production system», Computers \& Industrial Engineering, 53 ( I), pp. 196-198.

DURI, C; FREIN, Y. y LEE, H-S. (2000), «Performance evaluation and design of a CONWIP system with inspections», International Journal of Production Economics, 64 ( I-3), pp. 21 19-229.

FRAMINAN, J.M.; GONZÁLEZ-R, P.L. y RUIZ-USANO, R. (2003). «The Conwip production control system: review and research issues», Production Planning and Control, 14 (3), pp. 255-265.

GSTETTNER, S. y KUHN, H. (1996). «Analysis of production control systems Kanban and Conwip», International Journal of Production Research, 34 ( I I), pp. 3253-3274.

Superficie respuesta (WIP) para los sistemas Kanban y Conwip

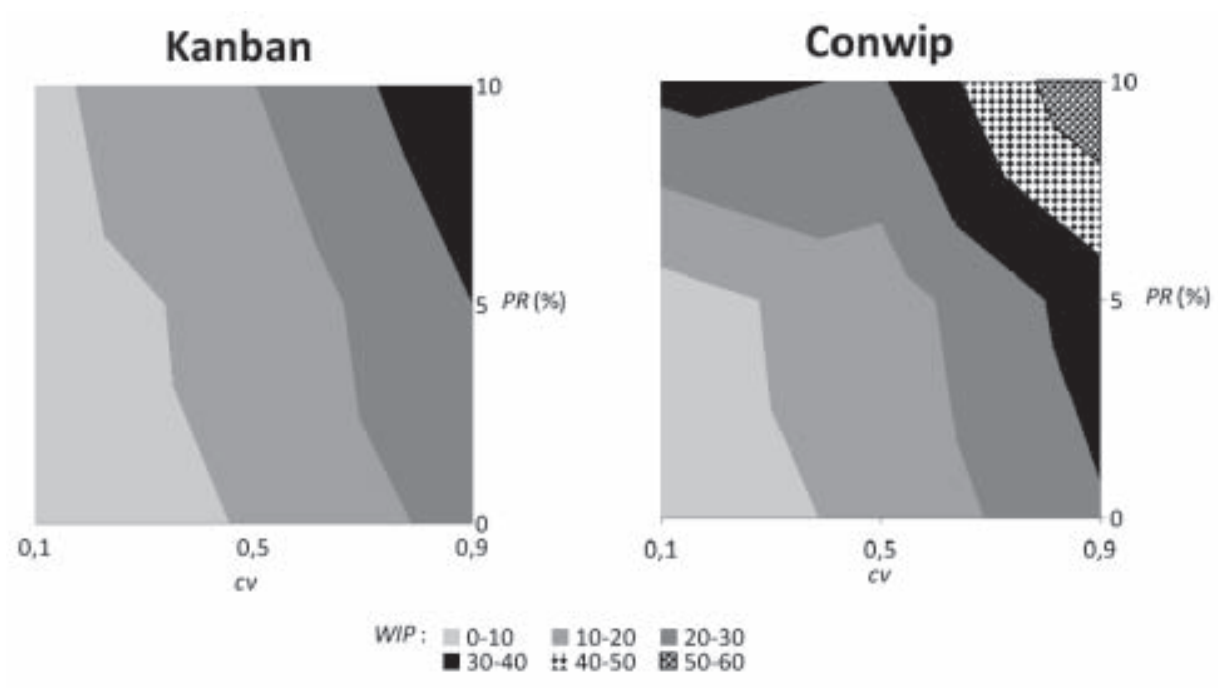


HOPP, W.J. (2007). Supply Chain Science, New York: McGraw-Hill.

HOPP, W.J. y SPEARMAN, M.L. (2000). Factory Physics: Foundations of Manufacturing Management, $2^{\text {nd }}$ ed. Burr Ridge, IL: Irwin/McGraw-Hill.

LAGE JUNIOR, M. y GODINHO FILHO. M. (20I0). «Variations of the Kanban system: Literature review and classification». International Journal of Production Economics, 125 ( I), pp. | 3-21.

MONTGOMERY, D.C. (2002). Diseño y análisis de experimentos, México D.F.: Ed. Limusa.

PYKE, D. F. y COHEN, M. A. (1990). «Push and Pull in Manufacturing and Distribution Systems», Journal of Operations Management, 9 ( I), pp. 24-43.
SPEARMAN, M.L.;WOODRUFF, D.L.y HOOP,W.J. ( 1 990). «Conwip: a pull alternative to Kanban», International Journal of Production Research, 28 (5), pp. 879-894.

SUGIMORI, Y.; KUSUNOKI, K.; CHO, F. y UCHIKAWA, S. (1977). «Toyota production system and kanban system materialization of just-in-time and respect-for-human system», International Journal of Production Research, I 5 (6), pp. 553-564.

UZSOY, R. y MARTIN-VEGA, L.A. ( 1990). «Modelling Kanban-Based Demand-Pull Systems: A Survey and Critique», Manufacturing Review, 3 (3), pp. I55-160.

XIAOBO, Z.; XU, D.; ZHANG, H. y HE, Q.M. (2007). «Modeling and analysis of a supply-assembly-store chain», European Journal of Operational Research, I76, pp.275294. 